\title{
Chirality-dependent G-band Raman intensity of carbon nanotubes
}

\section{Citation}

Saito, R., A. Jorio, J. H. Hafner, C. M. Lieber, M. Hunter, T. McClure, G. Dresselhaus, and M. S. Dresselhaus. 2001. "Chirality-Dependent G-Band Raman Intensity of Carbon Nanotubes." Physical Review B 64 (8). https://doi.org/10.1103/physrevb.64.085312.

\section{Permanent link}

http://nrs.harvard.edu/urn-3:HUL.InstRepos:41417433

\section{Terms of Use}

This article was downloaded from Harvard University's DASH repository, and is made available under the terms and conditions applicable to Other Posted Material, as set forth at http:// nrs.harvard.edu/urn-3:HUL.InstRepos:dash.current.terms-of-use\#LAA

\section{Share Your Story}

The Harvard community has made this article openly available.

Please share how this access benefits you. Submit a story.

Accessibility 


\title{
Chirality-dependent G-band Raman intensity of carbon nanotubes
}

\author{
R. Saito, ${ }^{1}$ A. Jorio, ${ }^{2}$ J. H. Hafner, ${ }^{3}$ C. M. Lieber, ${ }^{3}$ M. Hunter, ${ }^{4}$ T. McClure,${ }^{5}$ G. Dresselhaus, ${ }^{6}$ and M. S. Dresselhaus ${ }^{7}$ \\ ${ }^{1}$ Department of Electronic-Engineering, University of Electro-Communications, Tokyo, 182-8585 Japan \\ ${ }^{2}$ Department of Physics, Massachusetts Institute of Technology, \\ Cambridge, Massachusetts 02139-4307 \\ ${ }^{3}$ Department of Chemistry and Chemical Biology, Harvard University, Cambridge, Massachusetts 02138 \\ ${ }^{4}$ Department of Earth, Atmospheric and Planetary Sciences, Massachusetts Institute of Technology, \\ Cambridge, Massachusetts 02139-4307 \\ ${ }^{5}$ Center for Materials Science, Massachusetts Institute of Technology, \\ Cambridge, Massachusetts 02139-4307 \\ ${ }^{6}$ Francis Bitter Magnet Laboratory, Massachusetts Institute of Technology, Cambridge, Massachusetts 02139-4307 \\ ${ }^{7}$ Department of Electrical Engineering and Computer Science, Massachusetts Institute of Technology, \\ Cambridge, Massachusetts 02139-4307
}

(Received 10 September 2000; published 7 August 2001)

\begin{abstract}
The chirality-dependent G-band Raman intensity of single wall carbon nanotubes is calculated using a nonresonant theory for the Raman tensor. We obtain six or three intense Raman modes, respectively, for chiral or achiral nanotubes, whose relative intensities depend on the chiral angle of the nanotube. The longitudinal and transverse optical phonon modes in two-dimensional graphite become, respectively, transverse and longitudinal optical phonon modes in a one-dimensional nanotube. Confocal micro-Raman measurements of individual single wall carbon nanotubes show chirality-dependent spectra of the G-band intensity, as predicted by this theory.
\end{abstract}

DOI: 10.1103/PhysRevB.64.085312

PACS number(s): 78.30.Na, 78.20.Bh

\section{INTRODUCTION}

Carbon nanotubes ${ }^{1}$ have aroused world-wide interest in the quantum properties of electrons and phonons and in a variety of nanotube applications. ${ }^{2-4}$ Resonant Raman spectroscopy has provided a powerful tool for observing diameter-selective phonon modes in single wall carbon nanotubes $(\mathrm{SWNTs})^{4,5}$ and in multiwall carbon nanotubes $(\mathrm{MWNTs})^{6,7}$ containing different diameters within the sample. Among the 15 or 16 Raman active modes predicted by group theory, $2,3,8,9$ the radial breathing modes (RBM), and the tangential G-band modes are known to have strong Raman intensity in the lower $\left(100-450 \mathrm{~cm}^{-1}\right)$ and higher $\left(1500-1600 \mathrm{~cm}^{-1}\right)$ frequency regions, respectively, as firstorder Raman processes. In particular, the Raman-active $G$ band consists of two $A$, two $E_{1}$, and two $E_{2}$ phonon modes for chiral nanotubes, and of one $A_{1 g}$, one $E_{1 g}$, and one $E_{2 g}$ mode for achiral (armchair or zigzag) nanotubes, as predicted by group theory and phonon frequency calculations. $^{2,3,9}$ Thus we expect six or three G-band spectral features for chiral and achiral nanotubes, respectively, if all Raman-active modes have sufficient intensity to be observed and can be spectrally resolved.

The RBM frequency is inversely proportional to the diameter of the SWNT $d_{t}$ because the RBM frequency depends on the number of carbon atoms around the circumferential direction. ${ }^{10-12}$ As for the G-band phonon modes, different symmetry modes are split in frequency from one another by different amounts in the Raman spectra. The magnitude of the splitting of the G-band phonon modes increases with decreasing $d_{t}$ because of both a zone-folding effect ${ }^{13,14}$ and a curvature effect, ${ }^{15}$ each of which is operative on the phonon modes. Since the phonon frequencies of both the RBM and the $\mathrm{G}$ band depend mainly on $d_{t}$, previous theoretical works were performed mainly for achiral carbon nanotubes ${ }^{10}$ without considering any chirality dependence. However, direct calculation of the G-band frequencies for the $(17,0)$ zigzag nanotube showed that the lowest frequency component had $E_{2 g}$ symmetry, while the $E_{2 g}$ symmetry mode for the $(10,10)$ armchair nanotube ${ }^{10}$ was found to be the mode with the highest frequency. The present work shows that this difference in behavior is related to the chirality dependence of the G-band features discussed in this paper.

The symmetries of the intense Raman modes were observed in a recent Raman experiment for aligned MWNTs (Ref. 6) and aligned SWNTs. ${ }^{15,16}$ The experimental results are in good agreement with theory, ${ }^{10}$ when we consider both the nonresonant bond-polarization theory for the Raman intensity and the depolarization effect on the optical absorption. ${ }^{17-19}$ Furthermore, it is now possible to observe the Raman spectrum from an individual nanotube using polarized, ${ }^{19}$ surface enhanced, ${ }^{20,21}$ or confocal $^{22}$ microRaman techniques and directly observe the effects predicted in this paper. Moreover, we have succeeded in demonstrating a one-to-one assignment of the chiral indexes $(n, m)$ to each RBM frequency observed in a confocal micro-Raman experiment, using the theory of resonant transitions. ${ }^{22}$ We cannot, however, use this assignment to simultaneously interpret the RBM and G-band spectra, because the resonant condition for the RBM differs from that for the $G$ band by the G-band phonon energy $(\sim 0.2 \mathrm{eV})$. Thus, for a sample with several SWNTs within one light spot, a different nanotube might be resonant for the RBM as compared with the G band. Nevertheless, as shown in this paper, confocal micro-Raman observations of individual SWNTs show different G-band spectra from one SWNT to another. Thus a precise symmetry deter- 
mination of the G-band Raman-active modes for a chiral nanotube using theoretical considerations is necessary.

In this paper, we first present calculated results for the G-band Raman intensity of chiral carbon nanotubes, extending a model based on previous work. ${ }^{10}$ An interesting result is that for the case of chiral nanotubes, not three, but rather six phonon modes have sufficient Raman intensities to be observable, and furthermore the mode intensities also depend on nanotube chirality. This theoretical prediction is confirmed experimentally by the observation of chiralitydependent G-band Raman spectra for individual SWNTs, observed by use of confocal micro-Raman spectroscopy. ${ }^{22}$ These results provide valuable information for the identification of the chirality of a SWNT from a Raman experiment. In the next section, we show the methods used in the calculations and in the experiments. In Sec. III we show the calculated results of the G-band Raman intensity. In Sec. IV we present the observed G-band Raman spectra from an individual SWNT. The interpretation of these spectra is discussed in Sec. V along with further discussion of the model calculations. A summary of our results is given in Sec. VI.

\section{METHOD}

The Raman frequencies and intensities are calculated, respectively, using tight binding molecular dynamics (TBMD) methods and by a bond-polarization theory for the Raman tensor. $^{2,10}$ The limitations to this model are discussed in Sec. V.

The symmetries of the phonon modes are obtained by applying a projection operator for an irreducible representation with axially chiral symmetry $C_{N}$ to the calculated eigenvector of each phonon mode., ${ }^{2,3}$ In this paper the Raman intensity is calculated for the $V V$ polarization of the light by taking an average over random orientations of the nanotube axes, so that we obtain the total intensities for all modes corresponding to the $A, E_{1}$, and $E_{2}$ irreducible representations for the symmetry group $C_{N},{ }^{10}$ in which the depolarization effect cannot be seen in the calculated results. In the region of SWNT diameters, $1.30<d_{t}<1.40 \mathrm{~nm}$, we select eleven nanotube chiralities $(n, m)$ for our calculations: $(17,0),(17,1),(16,2),(16,3),(15,3),(15,4),(14,5)(13,7)$, $(12,7),(11,9)$, and $(10,10)$, and the chiral angles ${ }^{2}$ for these tubes $\theta=\arctan \{\sqrt{3} m /(2 n+m)\}$ lie between $0 \leqslant \theta \leqslant 30^{\circ}$, and are $0.0,2.8,5.8,8.5,9.0,11.5,14.7,20.1,21.4,26.7$, and $30.0^{\circ}$, respectively. The present calculation includes both metallic $\left(n-m=3 q\right.$ where $q$ is an integer $\left.{ }^{23}\right)$ and semiconducting $(n-m=3 q \pm 1)$ nanotubes.

In the confocal micro-Raman experiment, isolated SWNTs are prepared by a chemical vapor deposition method on a silicon-oxide $\left(\mathrm{SiO}_{2}\right)$ substrate containing nanometer iron catalyst particles. ${ }^{24}$ For a $4 \times 4 \mu \mathrm{m}^{2}$ atomic force microscopy (AFM) image of the sample, we identified 48 isolated SWNTs with lengths varying from $\sim 0.2 \mu \mathrm{m}$ to more than $2 \mu \mathrm{m}$. The distribution of SWNTs is not spatially homogeneous on the substrate, and 0 to 5 SWNTs can be found within a typical laser light spot, $1 \mu \mathrm{m}$ in diameter. Most of the nanotubes are individual SWNTs, but a few of them are occasionally entangled with each other, showing a "Y"

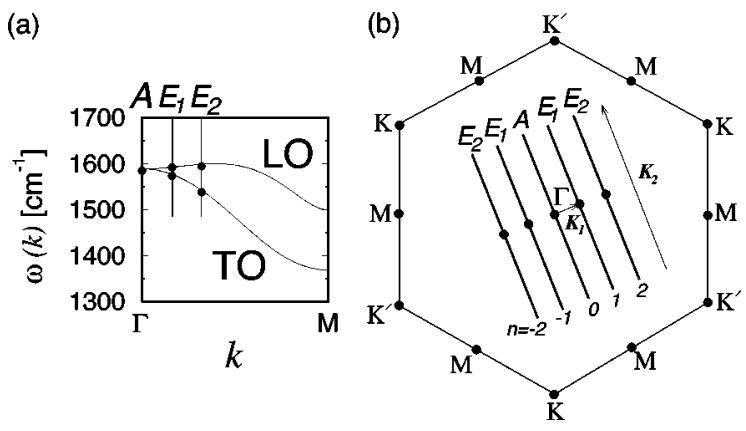

FIG. 1. (a) In-plane longitudinal and transverse optical (LO and TO) phonon dispersion relations of $2 \mathrm{D}$ graphite along the $\Gamma$ to $M$ directions. The zone folding of the $2 \mathrm{D}$ dispersion relations results in the $1 \mathrm{D}$ phonon modes for the $(6,6)$ armchair nanotube shown here. (b) Five solid lines in the hexagonal two-dimensional Brillouin zone of graphite represent part of the one-dimensional SWNT Brillouin zones which are relevant to the Raman intensity.

shape in some of the AFM images. The diameter distribution for 38 SWNTs observed by AFM (accuracy $\sim 0.2 \mathrm{~nm}$ ) can be fitted by a Gaussian distribution, with $d_{t}=1.76$ $\pm 0.73 \mathrm{~nm}$, which is almost the same as that for a previous substrate, ${ }^{22} d_{t}=1.85 \pm 0.62 \mathrm{~nm}$. Resonant Raman spectra from 100 to $1900 \mathrm{~cm}^{-1}$ were obtained from this sample/ substrate, using a Kaiser Optical Systems, Inc. Hololab 5000R: Modular Research Micro-Raman Spectrograph, with $25 \mathrm{~mW}$ power, and $E_{l}=785 \mathrm{~nm}=1.58 \mathrm{eV}$ solid state laser excitation. Both the incident and scattered beams were collected by optical fibers. The incident beam passes through a quarter-wave plate before being collected, so that no polarization of the light is expected in these observations.

The enhancement of a Raman signal is due to the resonance Raman effect in which an energy separation $E_{i i}$ between van Hove singularities (vH's) in the $i$ th valence band and the $i$ th conduction band, numbered starting from the Fermi energy, ${ }^{7,25}$ is close to the laser excitation energy $E_{l}$ $=1.58 \mathrm{eV}^{22}$ Therefore, although more than one SWNT (up to five SWNTs) can on average be illuminated within a laser spot, the probability of finding one resonant SWNT in a spot is very small, and several spots must be checked before we find a Raman signal from a resonant SWNT. ${ }^{22}$ Although the tube-surface interaction contributes to the Raman intensity, we did not observe a shift in the G-band frequencies in the experiment due to the tube-surface interaction. Thus we have applied the symmetry assignments determined for an aligned SWNT (Ref. 15) rope with a similar diameter to an analysis of the Raman spectrum of an isolated SWNT in the present paper.

\section{CALCULATED RESULTS}

In two-dimensional (2D) graphite, the in-plane longitudinal and transverse optical (LO and TO) phonons are degenerate at the $\Gamma$ point in the hexagonal, 2D Brillouin zone (BZ) as shown in Fig. 1(a) because of the hexagonal lattice symmetry. ${ }^{2}$ Zone-folding of the $2 \mathrm{D}$ BZ generates onedimensional phonon modes of a SWNT at the $\Gamma$ point of the 1D SWNT BZ from $k$ points which are separated by $n \mathbf{K}_{1}$, 


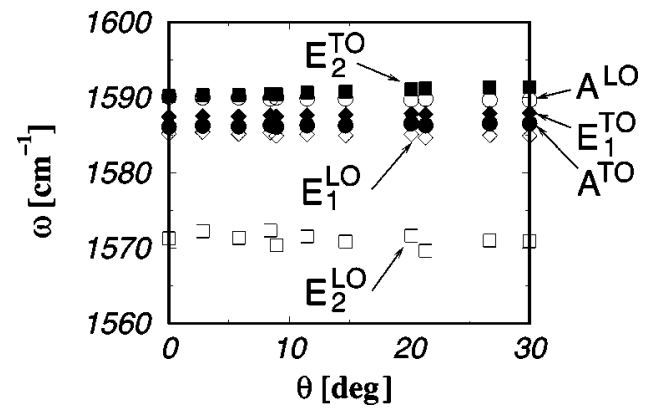

FIG. 2. The Raman frequencies of the G band of the SWNTs as a function of chiral angle $\theta$. The circle, diamond, and square symbols denote, respectively, $A, E_{1}$, and $E_{2}$ phonon modes, while the open and solid symbols denote, respectively, one-dimensional longitudinal and transverse optical (LO and TO) phonons.

$(n=0,1,2, \ldots)$ in the 2D BZ. These $k$ points are shown as dots on the five parallel lines in Fig. 1(b). Here $\mathbf{K}_{1}$ denotes a discrete unit vector in the $\mathrm{BZ}$ along the circumferential direction ${ }^{10}$ whose length is $2 / d_{t}$.

For an arbitrary $(n, m)$ nanotube, there are $N$ 1D BZ lines ${ }^{2}$ where $N=2\left(n^{2}+m^{2}+n m\right) / d_{R}$, and $d_{R}$ is the greatest common divisor of $(2 n+m)$ and $(2 m+n)$. We show only five lines in Fig. 1(b) for simplicity. For each dot in Fig. 1(b), we get two phonon frequencies which come from LO and TO phonon branches of 2D graphite, as shown in Fig. 1(a). The vector $\mathbf{K}_{2}$ in Fig. 1(b) denotes the reciprocal lattice vector of the 1D BZ whose direction corresponds to the nanotube axis. ${ }^{25}$ For the $1 \mathrm{D}$ phonon with an index $n$, there are $2 n$ nodes in the amplitude of each vibration around the circumferential direction, and the values of $n=0, \pm 1$ and \pm 2 correspond to the $A, E_{1}$, and $E_{2}$ phonon modes, respectively. Two orthogonal eigenvectors for the $E_{1}$ and $E_{2}$ phonon modes have amplitudes which behave as real quadratic functions $(x z, y z)$ and $\left(x y, x^{2}-y^{2}\right)$, respectively, on the $x y$ plane perpendicular to the nanotube axis. The other values of $n$ correspond to phonon modes at $k=0$ in the $1 \mathrm{D} \mathrm{BZ}$, which are not Raman active, and therefore do not contribute to the first-order Raman spectra.

Since the $n \mathbf{K}_{1}$ wave vectors coming from the $\Gamma$ points for the four dots with $n= \pm 1$ and \pm 2 in Fig. 1(b) correspond to propagating directions of the phonons in the $2 \mathrm{D} \mathrm{BZ}$, the displacements of the carbon atoms for the 2D LO and TO phonons are parallel and perpendicular to the propagating direction, respectively. However, the $n \mathbf{K}_{1}$ vectors are perpendicular to the nanotube axis direction $\left(\mathbf{K}_{2}\right)$. Thus, the $2 \mathrm{D} \mathrm{LO}$ and TO phonons of graphite become 1D TO and LO phonons of SWNTs, respectively. In general, LO modes have higher frequencies than TO modes in three-dimensional solids, but this is not the case for carbon nanotubes. Hereafter the labels of LO and TO are used for SWNTs without indicating their 1D dimensionality explicitly.

In Fig. 2 we plot the calculated frequencies of the G-band modes as a function of chiral angle. These calculations yield two $A$, two $E_{1}$ and two $E_{2}$ modes, which are denoted by circle, diamond, and square symbols, respectively. Open and solid symbols denote the 1D LO and TO phonons, respectively. The displacements of the carbon atoms for an eigen-

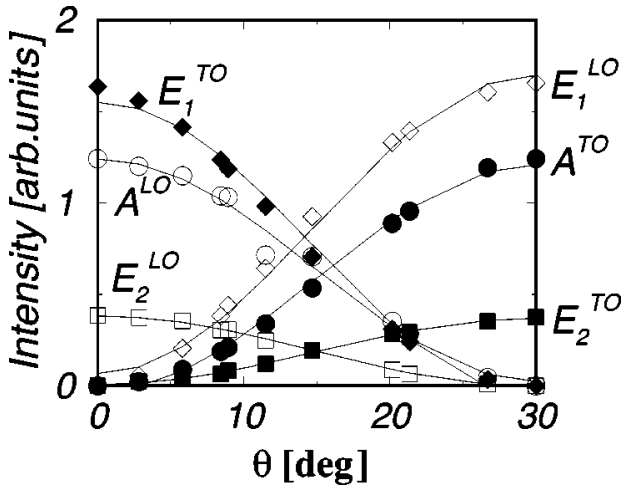

FIG. 3. The Raman intensity of the $G$ band as a function of chiral angle $\theta$. Open and solid symbols, respectively, denote the LO and TO phonons of the nanotubes. The calculated Raman intensities vs $\theta$ curves are all fitted to an angular dependence $B+C \cos (6 \theta)$.

vector of the SWNT phonon modes are always either parallel or perpendicular to the nanotube axis for the LO or TO phonon modes, respectively, for any symmetry type and for any chirality. This result comes from the curvature effect, which breaks the isotropic in-plane symmetry of the 2D graphite in-plane optic modes.

The TO mode frequencies of $E_{1}$ and $E_{2}$ symmetry are higher in SWNT's than the corresponding LO mode frequencies, since the 2D LO phonon branch of graphite has a higher frequency than the 2D TO phonon branch. As for the $A$ mode, the LO mode frequency is higher than that for the TO modes, because of the curvature effect, since we do not expect any zone-folding splitting for the $A$ modes as shown in Fig. 1(a).

The $G$-band mode frequencies do not depend on the chiral angle, and variations in the mode frequencies come from the diameter variation, since the phonon dispersion of the zonefolded 2D model is isotropic around the $\Gamma$ point in the 2D BZ. For nanotubes with $d_{t}=1.3-1.4 \mathrm{~nm}$, the highest and lowest calculated G-band frequencies correspond to the $E_{2}^{\mathrm{TO}}$ and $E_{2}^{\mathrm{LO}}$ modes, respectively. The large separation of the two $E_{2}$ mode frequencies mainly comes from the zone folding of the 2D LO and TO phonon branches, and the calculated value of the frequency separation for a $d_{t}=1.4 \mathrm{~nm}$ nanotube is around $20 \mathrm{~cm}^{-1}$, which is consistent with the work of Kasuya et al. ${ }^{13}$ For small diameter nanotubes (e.g., $d_{t}$ $=1.0 \mathrm{~nm}$ ), for which the curvature effect becomes dominant relative to the zone-folding effect, the calculated $A^{\mathrm{LO}}$ mode frequency becomes the highest. Furthermore, a gap between the $E_{2}^{\mathrm{LO}}$ mode frequency and that for other modes is found, since the 2D TO mode has a larger frequency dispersion than that for the 2D LO mode [see Fig. 1(a)].

In Fig. 3, we show calculated Raman intensities for the six phonon modes of the $G$ band as a function of the chiral angle $\theta$. The intensity for each mode is normalized by the number of carbon atoms in the unit cell $2 N$ since the Raman intensity is proportional to the number of carbon atoms in the unit cell, ${ }^{10,26}$ within the bond-polarization theory. An important aspect of the $\theta$ dependence of the mode intensities is the fact that the armchair nanotubes $\left(\theta=30^{\circ}\right)$ are expected to have Raman intensity for the $A_{1 g}^{\mathrm{TO}}, E_{1 g}^{\mathrm{LO}}$, and $E_{2 g}^{\mathrm{TO}}$ modes, 
(a) Zigzag

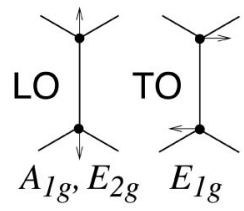

(b) Armchair

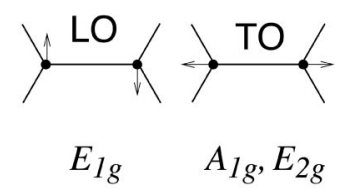

FIG. 4. The displacements of carbon atoms along the C-C bonds parallel and perpendicular to the nanotube axis for (a) zigzag and (b) armchair nanotubes, respectively. The labels denote the corresponding symmetries of the intense Raman modes.

while the zigzag nanotubes $\left(\theta=0^{\circ}\right)$ are expected to have Raman intensity for the $A_{1 g}^{\mathrm{LO}}, E_{1 g}^{\mathrm{TO}}$, and $E_{2 g}^{\mathrm{LO}}$ modes. In an armchair and zigzag nanotube, respectively, the $\mathrm{C}-\mathrm{C}$ bonds are perpendicular and parallel to the nanotube axis (see Fig. 4). When we examine the displacements of the carbon atoms for the intense Raman modes for armchair and for zigzag nanotubes, the displacements are perpendicular (bond bending modes) to the $\mathrm{C}-\mathrm{C}$ bonds for the $E_{1 g}$ modes, while the carbon atom displacements are parallel (bond stretching modes) to the C-C bonds for the $A_{1 g}$ and $E_{2 g}$ modes, as shown in Fig. 4. For large $\theta$, the $A^{\mathrm{TO}}, E_{1}^{\mathrm{LO}}$, and $E_{2}^{\mathrm{TO}}$ modes have relatively larger intensities, while the $A^{\mathrm{LO}}, E_{1}^{\mathrm{TO}}$, and $E_{2}^{\mathrm{LO}}$ modes are dominant for small $\theta$.

The calculated chirality dependence of the mode intensities varies monotonically from the armchair to the zigzag nanotube, as shown in Fig. 3. The mode intensities can be fitted by the angular dependence $B+C \cos (6 \theta)$ (where $B$ and $C$ are fitting parameters and the chiral angle $\theta$ is in units of radians). This clearly shows that the components of each vibration in the direction of the $\mathrm{C}-\mathrm{C}$ bonds (with threefold rotational symmetry) contribute to the Raman intensity, which has sixfold rotational symmetry with respect to $\theta$. In all cases, the calculated $E_{1}$ intensity is the largest, and the intensity ratio of the $A$ mode relative to that of the $E_{2}$ to $E_{1}$ modes, is independent of $\theta$. This result is obtained by averaging the Raman intensity over the nanotube axis directions relative to the direction of the polarized light. Although the $A$ phonon mode has a large intensity when the nanotube axis direction is parallel to the polarization, the dominant contribution to the total intensity comes from the large solid angle of nanotube directions perpendicular to the nanotube axis, where the $E_{1}$ modes have a large intensity. ${ }^{10}$

Within the bond polarization theory, the Raman intensity depends on the direction of the carbon atom displacements, not relative to the nanotube axis, but relative to the $\mathrm{C}-\mathrm{C}$ bonds. From a theoretical standpoint, since the Raman intensities for the TO modes seem to be larger than those for the LO modes, the bond polarization perpendicular to the nanotube axis contributes substantially to the Raman intensity. In fact, the RBM which has a strong Raman intensity corresponds to carbon atom displacements perpendicular (transverse mode) to the nanotube axis. Furthermore, when a SWNT of finite length has an open end, the phonon modes whose atomic displacements are parallel to the nanotube axis (longitudinal mode) have a very weak Raman intensity in the silent frequency region $\left(\sim 400-1000 \mathrm{~cm}^{-1}\right)$ for infinite

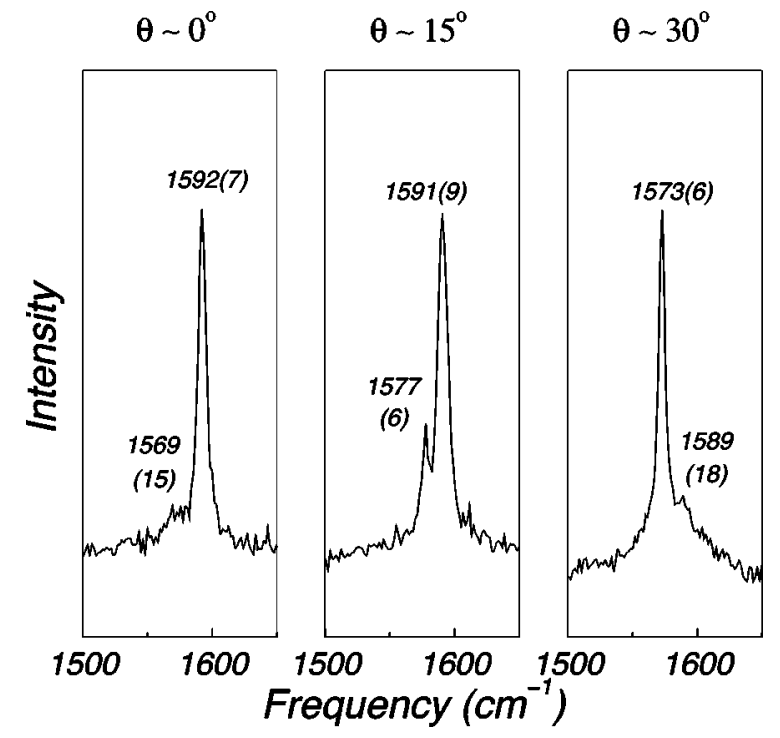

FIG. 5. Three G-band Raman spectra, each from an individual semiconducting SWNT, for three different light spots of the confocal micro-Raman experiment. The frequency (widths) of the peaks are displayed in $\mathrm{cm}^{-1}$.

SWNT's, ${ }^{26}$ in which the bond polarization makes a net contribution at the end of the nanotube. Thus, in accordance with Fig. 4, we might expect that the $E_{1 g}^{\mathrm{TO}}$ mode for zigzag nanotubes and the $A_{1 g}^{\mathrm{TO}}$ and $E_{2 g}^{\mathrm{TO}}$ modes for armchair nanotubes might have a larger intensity, based on the vibration direction of the carbon atoms. However, the depolarization effect, which is also important, shows a different angular dependence ${ }^{17,18}$ and this is discussed in Sec. V.

\section{G-BAND SPECTRA IN CONFOCAL MICRO-RAMAN SPECTROSCOPY}

Figure 5 shows the resonant Raman spectra from three different positions on the $\mathrm{Si} / \mathrm{SiO}_{2}$ substrate, each spot having one different SWNT that is in resonance with $E_{l}=1.58 \mathrm{eV}$. In our confocal micro-Raman experiment on individual single wall carbon nanotubes, we observe some G-band Raman spectra which exhibit only Lorentzian line shapes (as shown in Fig. 5), and are therefore assigned to semiconducting SWNTs, and some G-band spectra that have a broad Breit-Wigner-Fano (BWF) feature at about $1550 \mathrm{~cm}^{-1}$ (not shown), which we have assigned as coming from metallic SWNTs. ${ }^{27}$ Among the data observed from many isolated nanotubes, we restrict our discussion here to those spectra which exhibit only Lorentzian line shapes, since the present paper does not consider the interaction of the discrete phonon lines with an electron-related excitation continuum, ${ }^{28}$ which gives rise to the BWF line shape that is observed in metallic carbon nanotubes. ${ }^{5}$ As we discuss in the next section, the differences observed in the three spectra shown in Fig. 5 are due to the chirality dependence of the Raman intensity for the different symmetry modes within the $\mathrm{G}$ band in individual SWNTs. 


\section{DISCUSSION}

For a direct comparison between the experimental results shown in Fig. 5 and the predicted chirality dependence for the Raman intensity for different symmetry modes within the $\mathrm{G}$ band in SWNTs, it is first necessary to make a symmetry assignment of the modes observed in the spectra. The middle spectrum in Fig. 5 shows the usual G-band line shape, commonly observed in the resonant Raman measurements on SWNT bundle samples containing several semiconducting SWNTs (Refs. 4,5) in resonance with the incident or scattered photon. Two principal features are observed at 1591 and $1577 \mathrm{~cm}^{-1}$. Recent polarization Raman studies ${ }^{15}$ showed that those two features are formed by two unresolved modes having $A$ and $E_{1}$ symmetries, with the $A$ modes having the larger intensities. Two $E_{2}$ modes are expected to appear at lower $\left(\sim 1550 \mathrm{~cm}^{-1}\right)$ and higher $(\sim 1607$ $\mathrm{cm}^{-1}$ ) (Ref. 15) frequencies, but the $E_{2}$ modes are hardly seen in Fig. 5, which is consistent with the previous polarized Raman measurements on aligned semiconducting SWNTs for which the intensity of the $E_{2}$ modes was observed to be very small, and smaller than expected theoretically.

For the assignment of the TO and LO nature of these modes, we must consider the calculation discussed in Sec. III, and displayed in Fig. 2. The force constants used for the results in Sec. III fit well to the two-dimensional (2D) graphite energy dispersion relations, ${ }^{29,30}$ but it is known that the calculated RBM frequencies are $\sim 10 \%$ smaller than the observed ones for $d_{t}=1.4 \mathrm{~nm}$ SWNTs. ${ }^{22}$ As for the G-band frequencies, the present calculated values of the frequency separations between the G-band phonon modes with different symmetries fall in the $1570-1590 \mathrm{~cm}^{-1}$ range. The calculated range is, however, smaller than the spectral range $1550-1610 \mathrm{~cm}^{-1}$ of the experimental G-band features ${ }^{15,22}$ for semiconducting tubes with $d_{t} \simeq 1.4 \mathrm{~nm}$. This discrepancy arises because our calculation considers the curvature effect only through the nearest neighbor $\mathrm{C}-\mathrm{C}$ interaction, though the force constants in the TBMD calculations are taken up to fourth nearest neighbors. Thus, the present calculated results have some limitations in providing a quantitative analysis of the predicted mode frequencies and resonant Raman intensities. The experiments indicate that the curvature effect is underestimated and should introduce more splitting into the calculated frequencies. However, two important results do not seem to be affected by these limitations of the model: (1) the frequencies depend very little on chiral angle and (2) the frequency order of the six symmetry constituents displayed in Fig. 2 does not change with chiral angle. This agreement between theory and experimental observation allows a TO/LO assignment to be made for the peaks observed in Raman spectra, such as shown in Fig. 5.

Considering the experimental symmetry assignments for the two principal peaks, ${ }^{15}$ and the calculated mode frequencies discussed in Sec. III (see Fig. 2), we assign the peak observed at $1577 \mathrm{~cm}^{-1}$ to a superposition of the $A^{\mathrm{TO}}$ and the $E_{1}^{\mathrm{LO}}$ symmetry modes, while the peak observed at $1591 \mathrm{~cm}^{-1}$ is assigned to a superposition of the $A^{\mathrm{LO}}$ and the $E_{1}^{\mathrm{TO}}$ symmetry modes. Therefore, considering the theoretical predictions for the G-band Raman mode intensities discussed in Sec. III (see Fig. 3), the relative intensities between these two peaks should be chirality dependent.

The two spectra shown on the left and right sides of Fig. 5 come from two different isolated SWNTs on the $\mathrm{Si} / \mathrm{SiO}_{2}$ substrate. These two spectra clearly show an interchange in intensity between the two peaks at $\sim 1572$ and $\sim 1590 \mathrm{~cm}^{-1}$. These single nanotube spectra provide experimental evidence for chirality-dependent G-band Raman intensities for carbon nanotubes, which we here discuss in relation to the theoretical predictions in Sec. III.

From the left to the right experimental spectra in Fig. 5, we see that the intensity of the higher frequency peak $\left(A^{\mathrm{LO}}\right.$, $E_{1}^{\mathrm{TO}}$ symmetries) decreases and of the lower frequency peak ( $A^{\mathrm{TO}}, E_{1}^{\mathrm{LO}}$ symmetries) increases. This is exactly the behavior predicted for the G-band intensity dependence on chirality, as $\theta$ increases from $0^{\circ}$ to $30^{\circ}$ (see Fig. 3). Therefore, considering the calculated chirality dependence of the G-band mode intensities in Fig. 3, the left side spectra in Fig. 5 come from a SWNT with a low chiral angle ( $\theta$ close to $0^{\circ}$ ), while the spectra at the right side of Fig. 5 come from a SWNT with high chiral angle ( $\theta$ close to $30^{\circ}$ ). These differences in line shape provide experimental evidence for the predicted chirality dependence of the G-band intensity discussed in Sec. III.

For the chirality assignment of the spectrum in the center of Fig. 5, the nonresonant theory discussed in Sec. III would give a value of $\theta \sim 10^{\circ}$. However, it is important to remember that in a resonant Raman spectrum of a SWNT bundle sample of semiconducting nanotubes with different chiralities, ${ }^{15}$ the lower frequency peak $\left(\sim 1572 \mathrm{~cm}^{-1}\right)$ always gives a smaller Raman intensity relative to that for the higher frequency peak $\left(\sim 1590 \mathrm{~cm}^{-1}\right)$. This observed asymmetric intensity behavior for the peaks cannot be explained by the bond-polarization theory in Fig. 3, where an average over all chiral SWNTs would give an approximately equal intensity for the peaks at $\sim 1570 \mathrm{~cm}^{-1}\left(A^{\mathrm{TO}}\right.$ and $\left.E_{1}^{\mathrm{LO}}\right)$ and at $\sim 1590 \mathrm{~cm}^{-1}\left(A^{\mathrm{LO}}\right.$ and $\left.E_{1}^{\mathrm{TO}}\right)$.

In resonance Raman spectroscopy, the enhancement of the Raman intensity depends on the phonon symmetries. ${ }^{31}$ Especially in the case of carbon nanotubes, the depolarization effect for optical transitions may affect the different phonon symmetries in a different way, since the direction of the vibrations may be essential for the depolarization effect. Thus we do not use, in the analysis of the experimental spectra, the predicted relative intensities between different G-band phonon symmetries, as calculated by the bond-polarization theory. However, we do use, in the interpretation of experiments, the predicted Raman intensities for modes with the same symmetry but with different chirality, which is the subject of the present paper. For the three G-band symmetry modes $A, E_{1}$, and $E_{2}$, the atomic vibrations for the optical modes are similar to one another, except for the amplitude along the circumferential direction and for the difference between the LO and TO modes (as discussed further below). In this sense, the relative Raman intensities for the three modes would be similar to one another in the same phonon branch, if there were no depolarization effect. In contrast, the depo- 
larization effect in the optical absorption is essential for obtaining different enhancement factors in the resonant Raman process for each phonon mode in the $\mathrm{G}$ band. To calculate the resonant Raman intensity, it is therefore necessary to consider both (1) the electron-phonon interaction for each phonon mode and (2) the depolarization effect, and the calculation of both of these effects is beyond the scope of this paper.

Considering the depolarization effect in carbon nanotubes, the absorption and emission of light is suppressed when the light is polarized perpendicular to the tube axis. ${ }^{17-19}$ This polarization effect can explain the experimental observation that there are a few SWNTs which have a larger $A^{\mathrm{TO}}$ mode intensity than the $A^{\mathrm{LO}}$ modes. This means that, although the spectrum in the center of Fig. 5 does not exhibit similar intensities for the higher and lower frequency peaks, the chiral angle for this resonant SWNT should be close to $\theta$ $=15^{\circ}$. Combined with the fact that the RBM spectrum can be observed for a single nanotube, ${ }^{32}$ and that there is a homogeneous chirality distribution, we expect that the anisotropic optical absorption is important to explain that $A^{\mathrm{LO}}$ is generally larger intensity than $A^{\mathrm{TO}}$. To study the detailed resonant Raman intensity, we need to consider the electronphonon coupling constant ${ }^{33}$ which might also depend on the phonon symmetries.

Although general Raman theory says that the symmetry selection rules do no longer strictly apply in the resonant Raman regime, we do not actually observe any additional phonon modes in the resonant Raman experiment with different symmetries in the G-band frequency region, which are not predicted by nonresonant theory. ${ }^{6,15}$ We interpret this experimental result to suggest that the electron-phonon interaction might work similarly for the optical phonon modes, whose amplitudes are modulated along the circumferential direction. Since we observe in the Raman spectroscopy of isolated single wall carbon nanotubes that there are different Raman intensities for various features in the same G-band phonon frequency region (see, for example, Fig. 5 and the recent paper by $\mathrm{Yu}$ and $\mathrm{Brus}^{34}$ ) using the same laser energy but on different light spots (similar diameter tubes but with different chiralities), the theory presented here is able to explain most of the experimental observations associated with the G band for the Raman spectra of isolated SWNTs. Furthermore, the comparison between experiments and theory indicate where further improvements to the theory are particularly needed.

\section{SUMMARY}

We show in the present study both from theoretical and experimental work that the G-band intensity is chirality dependent. In particular, we give a clear view of the relationship between the symmetry of the G-band phonon modes and the Raman intensity from theory. From a confocal resonant micro-Raman measurement of isolated SWNTs, we discuss the important physics that is here involved, first from an experimental point of view and then as the experiments relate to the theory. The comparison between experiment and theory permits us to estimate the chiral angle $\theta$ for an isolated SWNT, thus contributing to improving the accuracy and uniqueness of the $(n, m)$ assignment of a SWNT observed by resonant Raman spectroscopy, ${ }^{15}$ and also in the identification of whether or not the RBM and the G-band features, observed in the Raman spectra from one light spot on the sample, come from the same resonant SWNT.

\section{ACKNOWLEDGMENTS}

The confocal micro-Raman experiment made use of the MRSEC Shared Facilities supported by the NSF under Grant No. DMR-9400334 and NSF Laser Facility Grant No. 9708265-CHE. The authors thank Professor M. A. Pimenta, Dr. A. G. Souza Filho, Dr. S. D. M. Brown and Mr. S. B. Cronin for discussions. R.S. acknowledges a Grant-in-Aid (No. 11165216 and 13440091) from the Ministry of Education, Japan and A.J. acknowledges financial support from CNPq Brazil. The MIT authors acknowledge support under NSF Grants No. DMR 98-04734, INT 98-15744, and INT 0000408 .
${ }^{1}$ S. Iijima, Nature (London) 354, 56 (1991).

${ }^{2}$ R. Saito, G. Dresselhaus, and M. S. Dresselhaus, Physical Properties of Carbon Nanotubes (Imperial College Press, London, 1998).

${ }^{3}$ M. S. Dresselhaus, G. Dresselhaus, and P. C. Eklund, Science of Fullerenes and Carbon Nanotubes (Academic Press, New York, 1996).

${ }^{4}$ M.S. Dresselhaus and P.C. Eklund, Adv. Phys. 49, 705 (2000).

${ }^{5}$ A.M. Rao, E. Richter, S. Bandow, B. Chase, P.C. Eklund, K.W. Williams, M. Menon, K.R. Subbaswamy, A. Thess, R.E. Smalley, G. Dresselhaus, and M.S. Dresselhaus, Science 275, 187 (1997).

${ }^{6}$ A.M. Rao, A. Jorio, M.A. Pimenta, M.S.S. Dantas, R. Saito, G. Dresselhaus, and M.S. Dresselhaus, Phys. Rev. Lett. 84, 1820 (2000).

${ }^{7}$ H. Kataura, Y. Kumazawa, Y. Maniwa, I. Umezu, S. Suzuki, Y.
Ohtsuka, and Y. Achiba, Synth. Met. 103, 2555 (1999).

${ }^{8}$ M.S. Dresselhaus, G. Dresselhaus, and R. Saito, Phys. Rev. B 45, 6234 (1992).

${ }^{9}$ D. Kahn and J.P. Lu, Phys. Rev. B 60, 6535 (1999).

${ }^{10}$ R. Saito, T. Takeya, T. Kimura, G. Dresselhaus, and M.S. Dresselhaus, Phys. Rev. B 57, 4145 (1998).

${ }^{11}$ S. Bandow, S. Asaka, Y. Saito, A.M. Rao, L. Grigorian, E. Richter, and P.C. Eklund, Phys. Rev. Lett. 80, 3779 (1998).

${ }^{12}$ M. Milnera, J. Kurti, M. Hulman, and H. Kuzmany, Phys. Rev. Lett. 84, 1324 (2000).

${ }^{13}$ A. Kasuya, Y. Sasaki, Y. Saito, K. Tohji, and Y. Nishina, Phys. Rev. Lett. 78, 4434 (1997).

${ }^{14}$ A. Kasuya, M. Sugano, T. Maeda, Y. Saito, K. Tohji, H. Takahashi, Y. Sasaki, M. Fukushima, Y. Nishina, and C. Horie, Phys. Rev. B 57, 4999 (1998).

${ }^{15}$ A. Jorio, G. Dresselhaus, M.S. Dresselhaus, M. Souza, M.S.S. 
Dantas, M.A. Pimenta, A.M. Rao, R. Saito, C. Liu, and H.M. Cheng, Phys. Rev. Lett. 85, 2617 (2000).

${ }^{16}$ H.D. Sun, Z.K. Tang, J. Chen, and G. Li, Solid State Commun. 109, 365 (1999).

${ }^{17}$ H. Ajiki and T. Ando, Physica B 201, 349 (1994).

${ }^{18}$ G.S. Duesberg, W.J. Blau, H.J. Byrne, J. Muster, M. Burghard, and S. Roth, Chem. Phys. Lett. 310, 8 (1999).

${ }^{19}$ G.S. Duesberg, I. Loa, M. Burghard, K. Syassen, and S. Roth, Phys. Rev. Lett. 85, 5436 (2000).

${ }^{20}$ K. Kneipp, H. Kneipp, P. Corio, S.D.M. Brown, K. Shafer, J. Motz, L.T. Perelman, E.B. Hanlon, A. Marucci, G. Dresselhaus, and M.S. Dresselhaus, Phys. Rev. Lett. 84, 3470 (2000).

${ }^{21}$ S. Lefrant, I. Baltog, M. Lamy de la Chapelle, M. Baibarac, G. Louarn, C. Journet, and P. Bernier, Synth. Met. 100, 13 (1999).

${ }^{22}$ A. Jorio, R. Saito, J.H. Hafner, C.M. Lieber, M. Hunter, T. McClure, G. Dresselhaus, and M.S. Dresselhaus, Phys. Rev. Lett. 86, 1118 (2001).

${ }^{23}$ R. Saito, M. Fujita, G. Dresselhaus, and M.S. Dresselhaus, Appl. Phys. Lett. 60, 2204 (1992).

${ }^{24}$ J. H. Hafner and C. M. Lieber, J. Phys. Chem. B 105, 743 (2001).
${ }^{25}$ R. Saito, G. Dresselhaus, and M.S. Dresselhaus, Phys. Rev. B 61, 2981 (2000).

${ }^{26}$ R. Saito, T. Takeya, T. Kimura, G. Dresselhaus, and M.S. Dresselhaus, Phys. Rev. B 59, 2388 (1999).

${ }^{27}$ M.A. Pimenta, A. Marucci, S.A. Empedocles, M.G. Bawendi, E.B. Hanlon, A.M. Rao, P.C. Eklund, R.E. Smalley, G. Dresselhaus, and M.S. Dresselhaus, Phys. Rev. B 58, R16 016 (1998).

${ }^{28}$ S.D.M. Brown, A. Jorio, P. Corio, M.S. Dresselhaus, G. Dresselhaus, R. Saito, and K. Kneipp, Phys. Rev. B 63, 155414 (2001).

${ }^{29}$ R.A. Jishi and M.S. Dresselhaus, Phys. Rev. B 45, 11305 (1992).

${ }^{30}$ R.A. Jishi, L. Venkataraman, M.S. Dresselhaus, and G. Dresselhaus, Chem. Phys. Lett. 209, 77 (1993).

${ }^{31}$ A.C. Albrecht, J. Chem. Phys. 34, 1476 (1961).

${ }^{32}$ A. Jorio, A. G. Souza Filho, G. Dresselhaus, M. S. Dresselhaus, R. Saito, J. H. Hafner, C. M. Lieber, F. M. Matinaga, M. S. S. Dantas, and M. A. Pimenta, Phys. Rev. B 63, 245416 (2001).

${ }^{33}$ E. Richter and K.R. Subbaswamy, Phys. Rev. Lett. 79, 2738 (1997).

${ }^{34}$ Z. Yu and L. E. Brus, J. Phys. Chem. 104, 1995 (2000). 